\title{
Conference Report \\ Spin Experimentation with Unpolarized Colliding Beams at the LHC
}

\author{
Sergey Troshin (1)
}

check for

updates

Citation: Troshin, S. Spin Experimentation with Unpolarized Colliding Beams at the LHC. Symmetry 2021, 13, 1886. https:// doi.org/ 10.3390/sym13101886

Academic Editor: Theodota Lagouri

Received: 14 September 2021

Accepted: 27 September 2021

Published: 6 October 2021

Publisher's Note: MDPI stays neutral with regard to jurisdictional claims in published maps and institutional affiliations.

Copyright: (C) 2021 by the authors. Licensee MDPI, Basel, Switzerland. This article is an open access article distributed under the terms and conditions of the Creative Commons Attribution (CC BY) license (https:/ / creativecommons.org/licenses/by/ $4.0 /)$.

\author{
NRC “Kurchatov Institute”-IHEP, 142281 Protvino, Russia; troshin@ihep.ru
}

\begin{abstract}
A brief recollection of the problems related to a significant hyperon polarization observed in $p p$-collisions is given with an emphasis on the general role of spin in the dynamics of hadron interactions. The old, unsolved problem of the observation of a significant hyperon polarization can provide new insights as a result of the measurements of energies at the LHC; in combination with other measurements, these can be used to tag the QGP formation in $p p$-collisions with colliding beams. Polarization studies in the processes of hyperon production do not require the use of polarized beams or targets and can be performed in the existing experimental environment at the LHC. Model predictions based on the chiral dynamics and pictures of the impact parameter are presented for the illustration of a possible dynamical mechanism that leads to a hyperon polarization.
\end{abstract}

Keywords: unpolarized beams; hyperon polarization; spin filtering

\section{Introduction}

The importance of spin measurements in studies of hadron interaction dynamics is well known. However, there are very few well-established experimental facts regarding the behavior and dependence of spin observables at high energies. In particular, the knowledge of the energy dependence of such observables is crucial, since it could clarify the role of the degrees of freedom of the spin with increases in energy. The LHC machine allows high-energy collisions to be recorded, and therefore, it could help in obtaining information on this subject. General wisdom could lead in wrong direction; e.g., the experiments at the LHC have discovered several unexpected collective effects in small systems, such as $p p$-interactions (cf. the reviews in $[1,2]$ and the references to the original papers therein).

A feasible experimental direction for spin studies with existing unpolarized beams in the collider mode can be the use of self-analyzing particle decays, the most well-known example of which is the weak decay of the $\Lambda$-hyperon (cf., e.g., [3]). To study the energy dependence of spin effects, it is most relevant to choose a respective reaction for which measurements have already been performed with the widest range of energy variations, namely, the measurements of the polarization of the aforementioned final $\Lambda$-hyperon in the inclusive reaction $p p \rightarrow \Lambda X$. It is also important to use an already-existing experimental environment to perform this taskto eliminate the need for extra expenses.

The most interesting and persistent long-term spin effect was observed in inclusive hyperon production in collisions of unpolarized hadron beams. A very significant polarization of $\Lambda$-hyperons was discovered more than four decades ago (cf. [3] and the references therein); experimentally, the process of $\Lambda$-production has been studied more extensively than any other hyperon production process. Therefore, we concentrate on the particular pattern of $\Lambda$-polarization. The spin structure of this particle is rather simple and is determined by the spin of a strange quark in the $S U(6)$-quark model.

Experimental results from measurements of hyperon polarization at different energies are widely known [3] - they are stable for a long time and are well documented. The polarization of $\Lambda$ produced in unpolarized inclusive $p p$-collisions is negative (it is perpendicular to the production plane and is directed opposite to the normal of this plane) and energyindependent. It increases linearly with $x_{F}$ at large transverse momenta $\left(p_{\perp} \geq 1 \mathrm{GeV} / \mathrm{c}\right)$, 
and for such values of transverse momenta, it is almost $p_{\perp}$-independent. A comprehensive review of the experimental situation is given in [3]. It should be noted that the above results are for hyperons, which appear to be protons' fragmentation products.

The recent RHIC data could imply a decrease in the energy of the global polarization of $\Lambda$ and could testify that the analog of the classical vorticity is a leading dynamical mechanism in the global strange quark polarization in nuclear collisions [4]. However, one should also take into account that the data in this case reflect the polarization measured with respect to the reaction (not production) plane. The explicit connection of these observables is not clear.

Perturbative QCD with the collinear factorization scheme leads to vanishing values of $\Lambda$-polarization $[5,6]$ and is in conflict with experimental data and observed trends. The inclusion of higher twist contributions allows one to obtain higher values for polarization but does not change the qualitative dependence $p_{\perp}^{-1}$ predicted for the region of large transverse momenta [7-9]. It is difficult to reconcile this decreasing dependence with the flat one observed in the data. The inclusion of parton internal transverse momenta ( $k_{\perp}$-effects) into the polarizing fragmentation functions also leads to a decreasing trend of polarization [10]. This only allows one to change the scale of the decrease in polarization.

The aim of this note is to provide arguments for measurements of the hyperon polarization at the energies of the LHC. For this purpose, we discuss the mechanism leading to a nondecreasing energy dependence of the transverse polarization of $\Lambda$ produced in $p p$-collisions. We also briefly present the experimental feasibility of such measurements and point out the role of polarization studies in the production of strangeness as a complementary tool for QGP detection in small systems. We refer the readers interested in this subject to a more detailed review [3] and recent paper [4] that provide some hints about the energy dependence of the global $\Lambda$-polarization in heavy ion collisions. The measurements of the polarization $p_{\perp}$-dependence are very interesting from the point of view of the perturbative QCD.

We discuss the colliding beam mode of the LHC and do not concern ourselves with the program of spin studies through the use of a polarized fixed target [11].

\section{Mechanism of the Strange Quark Polarization}

In the simple quark model, the $\mathrm{u}$ - and d-quarks in $\Lambda$ are coupled to the $S=0, I=0$ diquark. It is strange quark polarization that is responsible for the transverse polarization of $\Lambda$. Thus, to explain the significant $\Lambda$-polarization, the dynamical mechanism of the strange quark polarization should be developed. There were several proposals of explanations of the polarization of strange quarks that were produced in the collisions of unpolarized protons. Among them, one should note the mechanism of Thomas precession [12] and the Lund model [13]. Both explanations are semiclassical. There is another semiclassical mechanism based on chiral spin filtering. We briefly mention it as an example in order to stress that the measurement of hyperon polarization at the energies of the LHC is not senseless, since the set of mechanisms leading to a non-vanishing polarization of $\Lambda$ even when the collision energy increases is not empty.

We think that the polarization of strange quarks originates from a genuine nonperturbative QCD (cf., e.g., [14]). The kinematics of hyperon production are in favor of this assumption. There are two phenomena in the non-perturbative sector of QCD: the confinement and spontaneous breaking of chiral symmetry $(\chi \mathrm{SB})$. The corresponding scales are determined by the parameters $\Lambda_{Q C D}$ and $\Lambda_{\chi}$, respectively. Chiral $S U(3)_{L} \times S U(3)_{R}$ symmetry is spontaneously broken in the region of distances that lie in the range between these two scales. The $\chi \mathrm{SB}$ dynamics lead to the generation of quark masses and the appearance of quark condensates. They describe the transition of current into constituent quarks. Constituent quarks are considered to be quasiparticles, i.e., they are a superposition of bare valence quarks and quark-antiquark pairs, and their masses have a magnitude comparable to that of a hadron mass scale. A hadron is represented as a loosely bounded 
system of its constituent quarks. The direct result of he $\chi \mathrm{SB}$ dynamics is the appearance of Goldstone bosons (GBs).

The colliding particles (protons) in the collider mode are unpolarized. The absence of polarization means that the probabilities of the states with spin up or spin down are identical. The main idea of the mechanism is the filtering of the two initial spin states due to the different strengths of interactions [15]. This filtering acts as a polaroid and leads to nonzero polarization of the particles in the final state ( $\Lambda$ in particular). The mechanism of such filtering can be developed on the basis of a chiral quark model. Namely, we exploit a feature of the chiral quark model in which a constituent quark $Q_{\uparrow}$ with a transverse spin in the up direction can fluctuate into a Goldstone boson and another constituent quark $Q_{\downarrow}^{\prime}$ with its spin in the opposite direction, thus performing a spin-flip transition [16]:

$$
Q_{\uparrow} \rightarrow G B+Q_{\downarrow}^{\prime} \rightarrow Q+\bar{Q}^{\prime}+Q_{\downarrow}^{\prime} .
$$

To compensate the quark spin flip $\delta \mathbf{S}$, an orbital angular momentum $\delta \mathbf{L}=-\delta \mathbf{S}$ should be generated in the final state of the reaction (1). The presence of this orbital momentum $\delta \mathbf{L}$, in turn, implies a certain shift in the impact parameter value of the final quark $Q_{\downarrow}^{\prime}$ (which, in turn, is transmitted to the shift in the impact parameter of $\Lambda$ )

$$
\delta \mathbf{S} \Rightarrow \delta \mathbf{L} \Rightarrow \delta \mathbf{b}_{Q^{\prime}}
$$

Due to the different strengths of interactions with different values of the impact parameter, the processes of the transition into the spin-up and spin-down states will have different probabilities, which eventually leads to the nonzero polarization of $\Lambda$.

In the the case of $\Lambda$-polarization, the relevant transitions of the constituent quark $U$ (cf. Figure 1) are correlated with the shifts $\delta b_{S}$ in the impact parameter $b_{S}$ of the final strange quark, i.e.:

$$
\begin{aligned}
& U_{\uparrow} \rightarrow K^{+}+S_{\downarrow} \Rightarrow-\delta \mathbf{b}_{S} \\
& U_{\downarrow} \rightarrow K^{+}+S_{\uparrow} \Rightarrow+\delta \mathbf{b}_{S} .
\end{aligned}
$$

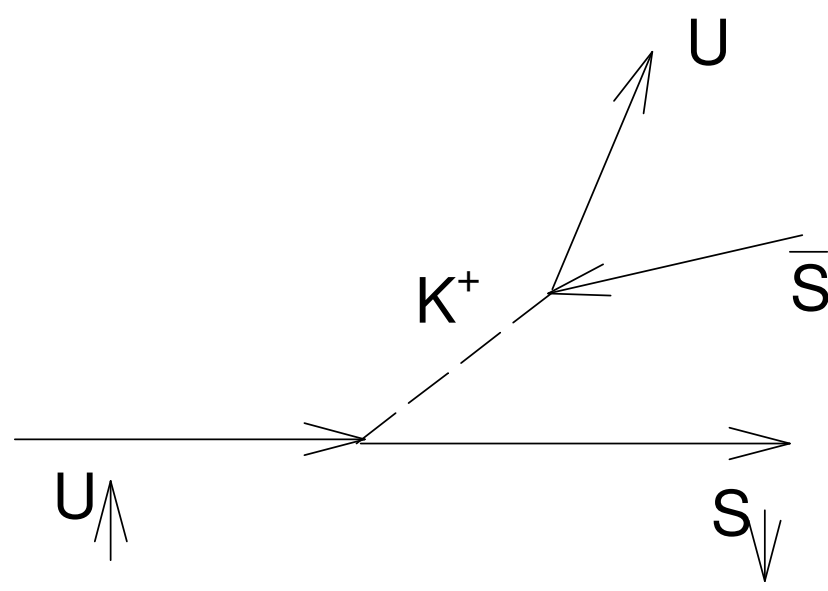

Figure 1. Transition of the constituent quark $U$ in the spin-up state to the strange quark in the spin-down state.

Relations (2) clarify the mechanism of spin-state filtering; when the shift in the impact parameter is $-\delta \mathbf{b}_{S}$, the interaction is stronger compared to the case in which the shift is $+\delta \mathbf{b}_{S}$, and the final $S$-quark (and $\Lambda$-hyperon) becomes negatively polarized. The mechanism of spin-state filtering considered here is suggested to be associated with the emission of Goldstone bosons by the constituent quarks. 
It is important to note here that the shift of $\mathbf{b}_{\Lambda}$ (the impact parameter of the final hyperon) is correlated with the shift of the impact parameter of the initial particles according to the relation between the impact parameters in multi-particle production [17]:

$$
\mathbf{b}=\sum_{i} x_{i} \mathbf{b}_{i}
$$

Let the variable $b_{\Lambda}$ be conjugated to the transverse momentum of $\Lambda$, but let the relations between functions that depend on the impact parameters $b_{i}$ be nonlinear. Since we are considering the production of $\Lambda$ in the fragmentation region, (i.e., at large $x_{F}$ ), the following approximate relation,

$$
b \simeq x_{F} b_{\Lambda}
$$

which results from Equation (3) (with an assumption of the smallness of Feynman $x_{F}$ for other secondary particles), is adopted.

The main point of this note is the energy dependence of the polarization, which can be checked experimentally. We note only that $\delta b_{S}$ (we assume that $\delta b_{S} \simeq \delta b_{\Lambda}$ ) can be connected with the radius of the quark interaction $r_{U \rightarrow S}^{\text {flip }}$ responsible for the transition $U_{\uparrow} \rightarrow S_{\downarrow}$ in which the quark spin and flavor are changed:

$$
\delta b_{S} \simeq r_{U \rightarrow S}^{f l i p} .
$$

To evaluate the dependence of the polarization on $x_{F}$ and $p_{\perp}$, we use the semiclassical correspondence between the transverse momentum and the impact parameter values. The energy and $p_{\perp}$-independent behavior of the polarization $P_{\Lambda}$ take place at large values of $p_{\perp}$ :

$$
P_{\Lambda}(s, \xi) \propto-x_{F} f_{U \rightarrow S}^{f l i p} M / \zeta
$$

This flat transverse momentum dependence results from the similar re-scattering effects for the different spin states. The numerical value of the polarization $P_{\Lambda}$ can be significant; there are no small factors in (5). We use the model from [18] in which $M$ is proportional to two nucleon masses, the value of parameter $\zeta \simeq 2$, and $r_{U \rightarrow S}^{f l i p} \simeq 0.1-0.2 \mathrm{fm}$. The above qualitative features of the dependence of polarization on $x_{F}, p_{\perp}$, and energy are in good agreement with the experimentally observed trends [3]. For example, Figure 2 demonstrates that the linear $x_{F}$ dependence is in good agreement with the experimental data in the fragmentation region $\left(x_{F} \geq 0.4\right)$ in which the model should work. Of course, the conclusion of the $p_{\perp}$-independence of $\Lambda$-polarization is a rather approximate one, and insignificant deviations from such behavior cannot be excluded.

The considered mechanism assumes the effective degrees of freedom and takes into account collective aspects of QCD dynamics. Together with unitarity, which is an essential ingredient of this approach, it allows one to obtain results for the dependence of polarization on kinematical variables in agreement with the experimental behavior of $\Lambda$-hyperon polarization, i.e., the linear dependence on $x_{F}$ and the flat dependence on $p_{\perp}$ at large $p_{\perp}$ in the fragmentation region are reproduced. These dependencies, together with the energyindependent behavior of polarization at large transverse momenta, are the straightforward consequences of this model.

We considered polarization in production in the fragmentation region. In the central region, where the correlations between the impact parameters of the initial particles and the impact parameters of the final particles are weak, polarization cannot be generated due to the chiral quark filtering mechanism. This is also valid for QGP production as a result of vacuum excitation. The transverse polarization of $\Lambda$ is expected to be zero, too [3]. Thus, the detection of vanishing transverse polarization might be associated with QGP production. Of course, the zeroing of transverse polarization is not sufficient to draw conclusions on QGP formation. This is important to note in view of the recent results of the ALICE measurements [19], where the data show for the first time that the yield of strangeness increases with multiplicity compared to the yield of pions. Such an 
enhancement at high multiplicities could be interpreted as a signal of QCP formation in small systems (cf. [19] and the references therein).
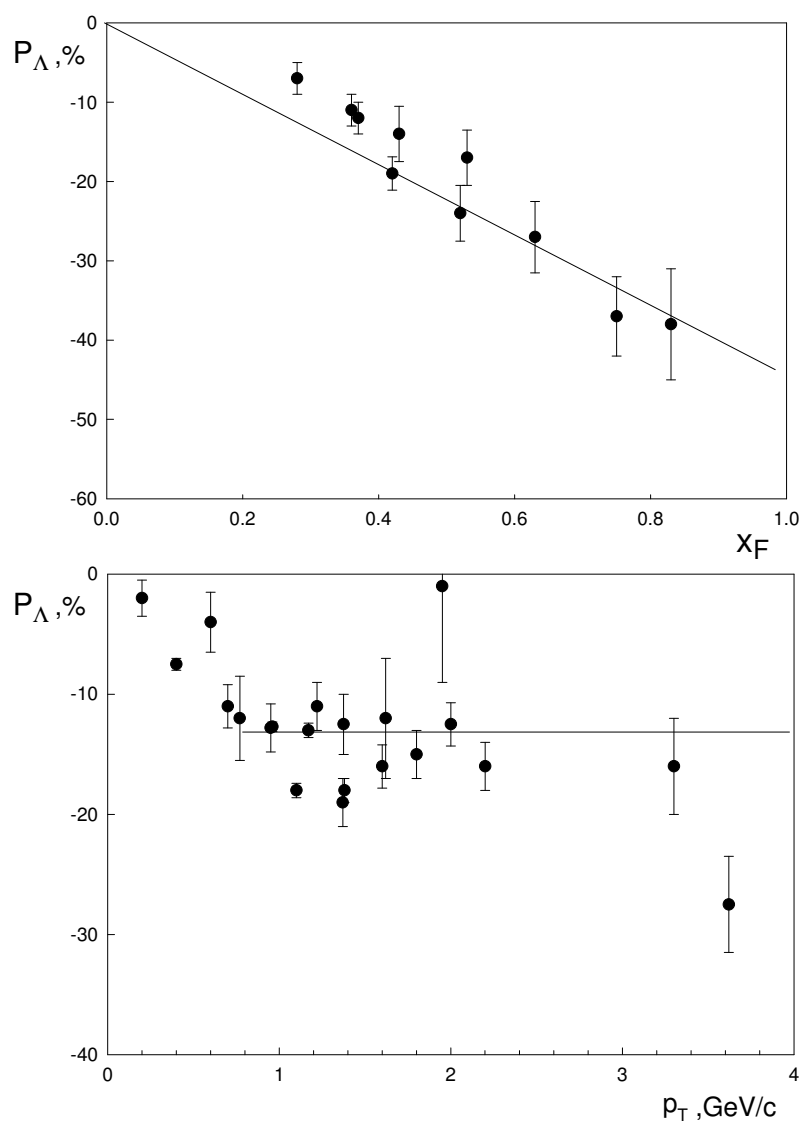

Figure 2. $x_{F}$ (top panel) and $p_{T}$ (bottom panel) dependencies of the $\Lambda$-hyperon polarization. See [3] for the experimental data sources.

The measurements performed at the LHC are also in favor of the above conclusion. Moreover, it is clear that since antiquarks are produced via spin-zero Goldstone bosons, we should expect transverse polarization $P_{\bar{\Lambda}} \simeq 0$. The chiral quark filtering is also relatively suppressed when compared to the direct elastic scattering of quarks, and therefore, it should not play a role in the reaction $p p \rightarrow p X$ in the fragmentation region, i.e., protons should be produced unpolarized. Indeed, these features appear in the experimental dataset.

We considered here the mechanism leading to the polarization of $\Lambda$ resulting from the fragmentation of a colliding proton. From this point of view, it seems rather natural to expect a significant polarization of $\Lambda$ in the process of the diffraction dissociation

$$
p+p \rightarrow \Lambda+K^{+}+X
$$

The measurements performed at ISR [20] are consistent with this observation.

\section{3. $\Lambda$-Polarization Measurements at the LHC}

The results of the previous section demonstrate that the spin effect studies at such high energies as those of the LHC are feasible; here, we mention the experimental feasibility of such measurements. These measurements are based on studies of the weak decay of $\Lambda$ into $p$ and $\pi^{-}$, which allows one to reconstruct $P_{\Lambda}$ from the angular distribution of the proton $d N / d \cos \theta_{p}$ produced as a result of this decay. This can be performed because this angular distribution is proportional to $1+\alpha_{\Lambda} P_{\Lambda} \cos \theta_{p}$, where $\theta_{p}$ is the angle between the nucleon's momentum and the axis of the hyperon's polarization. Plotting the distribution $d N / d \cos \theta_{p}$ against $\cos \theta_{p}$, the polarization can be obtained, since the value of the decay 
parameter $\alpha_{\Lambda}$ is known. Two-track events should be used to reconstruct weak $\Lambda \rightarrow p \pi^{-}$ decays, and it seems promising to use the T1 and T2 inelastic telescopes of the TOTEM experiment as a base for the relevant experimental setup.

The direction of $\Lambda$-polarization is directed along the normal to the production plane $\hat{\vec{n}}$ as a result of parity conservation in strong interactions. The unit vector $\hat{\vec{n}}$ is determined by the product

$$
\hat{\vec{n}}=\frac{\vec{p}_{b} \times \vec{p}_{\Lambda}}{\left|\vec{p}_{b} \times \vec{p}_{\Lambda}\right|}
$$

of the beam momenta $\vec{p}_{b}$ and $\vec{p}_{\Lambda}$ (momentum of $\Lambda$ ). In the central rapidity region, the transverse polarization of $\Lambda$ has a small value. However, the polarization of $\Lambda$ in diffraction dissociation processes is expected to be significant at the level $30-40 \%$ on the basis of experimental data extrapolations and estimations of simple semiclassical mechanisms. It is also important to check the energy-independence of the hyperon polarization observed at lower energies in order to bring a deeper understanding of the diffractive physics and the dependence on spin. One could note that the experience obtained at CERN ISR in the polarization measurements of $\Lambda$ could be helpful at the LHC, especially in the diffraction dissociation processes.

The measurements discussed above are also important for QGP detection. The importance of transverse polarization measurements has already been noted. An indirect way to measure the intensity of multi-strange baryon production was discussed earlier in [3] and is based on studies of the longitudinal polarization of $\bar{\Lambda}$ produced in the weak decay $\bar{\Xi} \rightarrow \bar{\Lambda}+\pi$ of the unpolarized $\bar{\Xi}$. This polarization arises due to the parity nonconservation in this weak decay process. As mentioned in [3], QGP formation might lead to a very significant longitudinal polarization of $\bar{\Lambda}$.

\section{Conclusions}

Hyperon polarization measurements at the LHC are important for studies of the energy dependence of spin effects. This would allow one to clarify the role of the degrees of freedom of the spin at such high energies, i.e., to get an answer to the question: Should we expect significant spin effects, or can one safely neglect the fermion nature of protons at such energies?

Finally, spin studies at the LHC are relevant tools for the strong sector of the testing of the Standard Model and are important for the determination of the region of applicability of perturbative QCD; in addition, they could serve as probes of QGP formation.

Funding: This research received no external funding.

Institutional Review Board Statement: Not applicable.

Informed Consent Statement: Not applicable.

Acknowledgments: The author is grateful to N.E. Tyurin for the comments and discussions.

Conflicts of Interest: The author declares no conflict of interest.

\section{References}

1. Troshin, S.M.; Tyurin, N.E. Collective effects in multiparticle production processes at the LHC. Int. J. Mod. Phys. A 2011, 26, 4703-4730. [CrossRef]

2. Schlichting, S.; Tribedy, P. Collectivity in Small Collision Systems: An Initial-State Perspective. Adv. High Energy Phys. 2016, 8460349. [CrossRef]

3. Panagiotou, A.D. $\Lambda^{0}$ Polarization in Hadron - Nucleon, Hadron - Nucleus and Nucleus-Nucleus Interactions. Int. J. Mod. Phys. 1990, 5, 1197-1266. [CrossRef]

4. Karpenko, I.; Becattini, F. Lambda polarization in heavy ion collisions: from RHIC BES to LHC energies. Nucl. Phys. A 2019, 982, 519-522. [CrossRef]

5. Kane, G.L.; Pumplin, J.; Repko, W. Transverse Quark Polarization in Large $p_{T}$ Reactions, $e^{+} e^{-}$Jets, and Leptoproduction: A Test of QCD. Phys. Rev. Lett. 1978, 41, 1689-1692. [CrossRef]

6. Dharmaratna, W.G.D.; Goldstein, G.R. Single quark polarization in quantum chromodynamics subprocesses. Phys. Rev. D 1996, 53, 1073-1086. [CrossRef] [PubMed] 
7. Efremov, A.V.; Teryaev, O.V. On Spin Effects in Quantum Chromodynamics. Sov. J. Nucl. Phys. 1982, 36, $140-147$.

8. Qiu, J.; Sterman, G. Single transverse spin asymmetries in hadronic pion production. Phys. Rev. D 1999, 59, 014004. [CrossRef]

9. Kanazawa, Y.; Koike, Y. Polarization in hadronic Lambda hyperon production and chiral odd twist-Three quark distribution. Phys. Rev. D 2001, 64, 034019. [CrossRef]

10. Anselmino, M.; Boer, D.; D’Alesio, U.; Murgia, F. $\Lambda$ polarization in unpolarized hadron reactions. Czech. J. Phys. 2001, 51 (Suppl. S1), A107-A113. [CrossRef]

11. Hadjidakis, C.; Anselmino, M.; Arnaldi, R.; Brodsky, S.J.; Chambert, V.; Da Silva, C.; Didelez, J.P.; Echevarria, M.G.; Ferreiro, E.G.; Fleuret, F.; et al. Physics opportunities with a fixed target experiment at the LHC (AFTER@LHC). In Proceedings of the XVII International Workshop on Polarized Sources, Targets and Polarimetry, Kaist, Korea, 16-20 October 2017; PoS PSTP2017 (2018) 035 .

12. DeGrand, T.A.; Miettinen, H.I. Models for polarization asymmetry in inclusive hadron production. Phys. Rev. D 1981, 24, 2419-2427. [CrossRef]

13. Andersson, B.; Gustafson, G.; Ingelman, G.; Sjöstrand, T. Parton Fragmentation and String Dynamics. Phys. Rep. 1983, 97, 31-145. [CrossRef]

14. Troshin, S.M.; Tyurin, N.E. Strangeness in constituent quarks and one spin asymmetry in inclusive phi meson production. Phys. Lett. B 1995, 355, 543-547. [CrossRef]

15. Troshin, S.M.; Tyurin, N.E. Chiral quark filtering mechanism of hyperon polarization. arXiv 2015, arXiv:hep-ph/0501004.

16. Cheng, T.P.; Li, L.-F. Why naive quark model can yield a good account of the baryon magnetic moments. Phys. Rev. Lett. 1998, 80, 2789-2792. [CrossRef]

17. Webber, B.R. The Impact Parameter Structure of Multiparticle Production. Nucl. Phys. B 1975, 87, 269-317. [CrossRef]

18. Troshin, S.M.; Tyurin, N.E. Flat transverse momentum dependence of the single-spin asymmetry in inclusive neutral pion production. Phys. Rev. D 2013,88, 017502. [CrossRef]

19. Adam, J.; Adamova, D.; Aggarwal, M.M.; Rinella, G.A.; Agnello, M.; Agrawal, N.; Ahammed, Z.; Ahmad, S.; Ahn, S.U.; Aiola, S.; et al. (ALICE Collaboration). Enhanced production of multi-strange hadrons in high-multiplicity proton-proton collisions. arXiv 2017, arXiv:1606.07424v2.

20. Erhan, S.; Lockman, W.; Medinnis, M.; Meyer, T.; Rander, J.; Schlein, P.; Webb, R.; Boehm, A.; Foeth, H.; Staude, A. $\Lambda^{0}$ Polarization in Proton Proton Interactions at $\sqrt{s}=53 \mathrm{GeV}$ and $62 \mathrm{GeV}$. Phys. Lett. B 1979, 82, 301-304. [CrossRef] 